\title{
Integration of 5G Networks and Internet of Things for Future Smart City
}

\author{
Bo Rong $\mathbb{D}^{1},{ }^{1}$ Shuai Han $\left(\mathbb{D},{ }^{2}\right.$ Michel Kadoch $\left(\mathbb{D},{ }^{3}\right.$ Xi Chen $\mathbb{D D}^{4}$ and Antonio Jara ${ }^{5}$ \\ ${ }^{1}$ Communications Research Centre Canada, Ottawa, ON, Canada \\ ${ }^{2}$ Department of Electronics and Communication Engineering, Harbin Institute of Technology, Harbin, Heilongjiang, China \\ ${ }^{3}$ Department of Electrical Engineering, Ecole de Technologie Superieure, Montreal, QC, Canada \\ ${ }^{4}$ Flatiron Institute, Simons Foundation, New York, NY, USA \\ ${ }^{5}$ University of Applied Sciences of Western Switzerland-HES SO Valais, Sierre, Switzerland \\ Correspondence should be addressed to Bo Rong; bo.rong@canada.ca
}

Received 7 November 2019; Accepted 7 November 2019; Published 12 February 2020

Copyright ( $) 2020$ Bo Rong et al. This is an open access article distributed under the Creative Commons Attribution License, which permits unrestricted use, distribution, and reproduction in any medium, provided the original work is properly cited.

The applications of IoT will proliferate in all sorts of devices, contributing to the infrastructure of smart city. These devices from different city sectors will cover every corner of the society, which calls for city-wide networks with ubiquitous accessibility to retrieve and deliver the produced data. Envisioning this demand, $5 \mathrm{G}$ has extended its mission to communicate things more than just people. The resulting $5 \mathrm{G}$ IoT contributes to the prosperity of the smart city ecosystem by allowing entities, big or small, to set up IoT services without the need to implement their own network facilities, compared with solutions, e.g., LoRa and SigFox. It is foreseeable plenty of smart city services will be running over $5 \mathrm{G}$, pushing forward the integration of $5 \mathrm{G}$ and IoT. This trend, in turn, is going to impose unprecedented challenges on the on-building $5 \mathrm{G}$ mobile network and impact the normative work of $5 \mathrm{G}$.

This special issue thus aims at bringing together the state-of-the-art innovations, research activities, and standardization updates related to the integration of $5 \mathrm{G}$ networks and IoT, in a bid to help both academic and industrial research communities understand the recent research advances and emerging technologies.

In the paper titled "Practical Aspects for the Integration of 5G Networks and IoT Applications in Smart Cities Environments," the authors investigate a number of practical issues related to $5 \mathrm{G}$-based IoT applications, including the need for small cells, the transmission issues at millimeter wave frequencies, building penetration issues, and the need for distributed antenna systems. To meet the special interest in smart cities environments, this work also presents a brief introduction to pre-5G IoT technologies, such as NB-IoT and LTE-M.

The paper titled "Co-Channel Coexistence Analysis between 5G IoT System and Fixed-Satellite Service at $40 \mathrm{GHz}$ " presents a promising way to successfully operate the fifth generation (5G) system with Internet of Things (IoT) in potential mmWave spectrum bands. The authors investigate the intelligent cochannel coexistence between the $5 \mathrm{G}$ IoT system and the fixed-satellite service (FSS) system at $40 \mathrm{GHz}$. The simulation results reveal that interference from the $5 \mathrm{G}$ IoT system into the FSS ground stations can be kept below the protection threshold by considering different deployment parameters, such as antenna patterns, height of Earth station (ES), and separation distance.

The paper titled "Radar-Assisted UAV Detection and Identification Based on 5G in the Internet of Things" proposes a radar-assisted positioning method for unmanned aerial vehicles (UAVs) based on $5 \mathrm{G}$ millimeter waves. The authors employ high-resolution range profile (HRRP), micro-Doppler characteristics, and the sinusoidal frequency modulation (SFM) parameter optimization method, respectively, to obtain the UAV location in the detection zone, identify the UAVs, extract the number and speed information of the UAV rotor, and separate multiple UAVs. The simulation results show that the proposed radar detection method is well suited for UAV detection and identification and provides a valid GPS-independent method for UAV tracking. 
The paper titled "Performance Analysis for Downlink MIMO-NOMA in Millimeter Wave Cellular Network with D2D Communications" develops closed-form expressions for the outage probability and ergodic capacity in downlink MIMO-NOMA mmWave cellular network with D2D communications considered. The influencing factors of performance, such as transmission power and antenna number, are analyzed with a conclusion that higher transmission power and more antennas in the base station can decrease the outage probability and enhance the ergodic capacity of NOMA.

The paper titled "Dynamic Traffic Prediction with Adaptive Sampling for 5G HetNet IoT Applications" suggests an improved Call Session Control Function (CSCF) scheme, in which the improved CSCF server contains additional modules to facilitate IoT traffic prediction and resource reservation. The authors develop a compressed sensing based linear predictor to catch the traffic patterns. The proposed CSCF scheme can forecast the traffic load with high accuracy but low sampling overhead.

In the paper titled "A Hybrid Predictive Strategy Carried through Simultaneously from Decision Space and Objective Space for Evolutionary Dynamic Multiobjective Optimization," the authors propose a hybrid prediction strategy carried through from both decision space and objective space (DOPS), in order to handle all kinds of optimization problems. The prediction in decision space is based on the center point, and the prediction in objective space is based on CTI. In addition, a kind of memory method is added to handle the problems with periodic changes, and a selfadaptive diversity maintenance method is adopted to compensate for the inaccuracy of the prediction in particularly complex problems. The experimental results show that DOPS is effective in dynamic multiobjective optimization.

\section{Conflicts of Interest}

The editors declare that they have no conflicts of interest regarding the publication of this special issue.

Bo Rong

Shuai Han

Michel Kadoch

Xi Chen

Antonio Jara 